\title{
Antimicrobial resistance trends in bloodstream infections at a large teaching hospital in China: a 20-year surveillance study (1998-2017)
}

Lei Tian', Zhen Zhang ${ }^{2}$ and Ziyong Sun ${ }^{1 *}$

\begin{abstract}
Background: Bacterial bloodstream infections (BSIs) cause high morbidity and mortality worldwide in humans, but the pathogenic spectrum varies from region to region. Long-term monitoring of the pathogenic spectrum and changes in bacterial antibiotic resistance is hugely important for effective clinical therapy and infection control. This study examined the data for BSIs in Tongji Hospital, one of the largest teaching hospitals in China, in an attempt to gain better understanding of bacterial antibiotic resistance in China, focusing on central China.

Methods: Data from Tongji Hospital for a 20-year period (1998-2017) were used for a retrospective analysis to understand the pathogenic spectrum of BSIs and the changes occurring in antimicrobial resistance in central China. The disk diffusion and $\mathrm{E}$ test methods were used for antimicrobial susceptibility testing according to Clinical \& Laboratory Standards Institute methodologies, and the data were analyzed by WHONET 5.6 software.

Results: The isolated pathogens mainly came from hospitalized patients not treated in intensive care units (ICUs), and accounted for $81.5 \%$ of the total (9130/11200). The most common Gram-negative and Gram-positive bacterial BSI-causing pathogens were Escherichia coli and Staphylococcus aureus, respectively. The detection rate for methicillin-resistant S. aureus (MRSA) in the hospitalized non-ICU patients increased from 8.4\% in 1998-2002 to 63\% in 2013-2017, while the detection rate for carbapenem-resistant (CR) Klebsiella pneumoniae was below 5\% in 19982012 but increased to 34.9\% in 2013-2017. In contrast, worryingly, the detection rate for CR K. pneumoniae in ICU patients increased from 0\% in 2013 to 75\% in 2016. E. coli displayed the highest sensitivity rates to imipenem, meropenem and amikacin, all of which were $>90 \%$, followed by cefoxitin at $>80 \%$, and cefoperazone/sulbactam at $>70 \%$. K. pneumoniae isolates were most sensitive to imipenem, meropenem and amikacin antibiotics, with sensitivity rates exceeding $60 \%$. S. aureus isolates were most sensitive to vancomycin, teicoplanin and trimethoprim/ sulfamethoxazole, with sensitivity rates exceeding $90 \%$.
\end{abstract}

Conclusions: BSIs caused by CR K. pneumoniae clearly posed a severe challenge to infection control and treatment of ICU and non-ICU patients in this retrospective study, while MRSA was an issue for non-ICU patients.

Keywords: Bloodstream infections, Bacterial pathogens, Antimicrobial resistance, Retrospective analysis, Patients, Central China

\footnotetext{
* Correspondence: zysun@tjh.tjmu.edu.cn

${ }^{1}$ Department of Clinical Laboratory, Tongji Hospital, Tongji Medical College,

Huazhong University of Science and Technology, Wuhan, Hubei Province,

China

Full list of author information is available at the end of the article
}

(c) The Author(s). 2019 Open Access This article is distributed under the terms of the Creative Commons Attribution 4.0 International License (http://creativecommons.org/licenses/by/4.0/), which permits unrestricted use, distribution, and reproduction in any medium, provided you give appropriate credit to the original author(s) and the source, provide a link to the Creative Commons license, and indicate if changes were made. The Creative Commons Public Domain Dedication waiver (http://creativecommons.org/publicdomain/zero/1.0/) applies to the data made available in this article, unless otherwise stated. 


\section{Background}

Bloodstream infections (BSIs) inflict high morbidity and mortality on people from both developed and developing countries in the world. Analyzing the pathogenic spectrum of BSIs and the changes occurring in the antimicrobial resistance patterns of pathogenic bacteria from the data accumulated over many years is very important for clinicians and for infection control. However, the pathogenic spectrum and bacterial resistance patterns of BSIs reportedly differ in the different regions of the world. For example, in Malawi, Africa, the most common pathogens causing BSIs were found to be nontyphoidal salmonella, Salmonella typhi, and Streptococcus pneumoniae in an analysis of accumulated data over 19 years (1998-2016) [1]. In contrast, data from the European Antimicrobial Resistance Surveillance Network (EARS-Net, formerly EARSS, which includes 198 laboratories in 22 European countries) from 2002 to 2009 indicated that Escherichia coli and Staphylococcus aureus were the most common BSI-causing pathogens [2]. Similarly, the surveillance network data (KorGLASS) from South Korea in 2016-2017 showed that $E$. coli and Staphylococcus aureus were the most common BSI-causing pathogens [3], whereas the data for Japan showed that E. coli, Staphylococcus aureus, Streptococcus spp. and Klebsiella spp. were the most common BSIcausing pathogens [4].

The data for BSIs in China are very limited. Hence, in the present study, we analyzed the BSI data for Tongji Hospital in Wuhan, the capital city of Hubei Province in central China. Tongji Hospital is one of the largest Chinese teaching hospitals in China and from 2018 this hospital supplies three sub-districts with 6000 beds in total. Tongji Hospital was the first Asian hospital to pass the Kooperation für Transparenz und Qualiät im Gesundheitswesen certification in Germany and the Department of Laboratory Medicine was one of the first laboratories in China to pass both the International Organization for Standardization 15, 189 and College of American Pathologists (CAP) certification in the USA. At the same time, Tongji Hospital also acted as the central network hospital for the Antimicrobial Resistance Surveillance System (HBRASS) in Hubei Province. The data from Tongji Hospital are, therefore, representative of the bacterial resistance patterns in central China. Our previous studies from HBRASS from 2014 to 2016 showed that the most common BSI-causing pathogens in Hubei Province were E. coli, Staphylococcus aureus and K. pneumoniae [5]. The data accumulated for Tongji Hospital over the last 20 years (1998-2017) is a very valuable and meaningful resource for understanding the pathogenic spectrum and bacterial resistance in BSIs in China, especially central China.

\section{Materials and methods}

\section{Study design and procedures}

This study was a retrospective analysis of all the data on blood cultures accumulated by the Microbiology Laboratory from the Department of Laboratory Medicine of Tongji Hospital from 1998 to 2017. Blood culture specimens from all patients including adults and children in Tongji Hospital were sent to the Microbiology Laboratory for testing. Blood culture was performed when the patient had the following clinical symptoms or signs [6]: 1. The patient's body temperature was higher than $38^{\circ} \mathrm{C}$ or lower than $36^{\circ} \mathrm{C}$, and was also the most common clinical situation for ordering blood culture. 2 . The peripheral blood cell count showed an increase in white blood cells (WBCs) above $10 \times 10^{9} / \mathrm{L}$ or a decrease in the WBC count below $4 \times 10^{9} /$ L. 3. A patient's respiratory rate exceeded 20 breaths per minute or the arterial partial pressure of carbon dioxide was under $32 \mathrm{mmHg}$. 4. The patient's heart rate exceeded 90 beats per minute. 5. The patient developed chills, coma, skin and mucosal bleeding, hypotension or multiple organ dysfunctionality. 6. The laboratory tests showed increases in inflammatory response indicators (e.g., $\mathrm{C}$ reactive protein, procalcitonin, and a positive $\mathrm{G}$ test, among others). Blood sample volumes were $8-10 \mathrm{ml}$ per bottle for adult patients and 2-5 $\mathrm{ml}$ per bottle for children. For adult patients, each set included an aerobic bottle and an anaerobic bottle, but only an aerobic bottle was used for children. The instruments used for blood culturing were purchased from the BD Company (product models, 9120, 9240 and FX400 ) and the BioMerier Company (3D instrument system). Samples from adults were analyzed on the BD and BioMerier systems; samples from children were analyzed on the BioMerier system.

The incubation period for common bacteria was 5 days, and 14 days for fungi. When it was suspected that the pathogen was a slow-growing bacterium like Brucella, the incubation period was extended appropriately. If a positive alarm occurred in the blood culture instrument, smear, stain, microscopic examination and transfection were carried out. When false positive results were found via blood smears, microscopic examination, or observing the growth curve from the instrument, the blood culture bottle would be returned to the instrument within one hour to continue the culture. Strain identification was carried out biochemically, using the automatic identification system (Vitek-2-compact, BioMerier Products) and/or the IVD-MALDI Biotyper (Bruker, Germany). Antimicrobial susceptibility tests were carried out according to Clinical \& Laboratory Standards Institute (CLSI) approved procedures using disk diffusion and $\mathrm{E}$ test methods.

All laboratory operations were carried out in strict accordance with the standardized operating procedures 
of the hospital departments. The laboratories maintained good indoor quality control and external quality assessment was conducted. Before each batch of blood culture bottles was tested, quality control verification involved the use of standard strains (ATCC25923, 25922, 27852, 49619, and 49247). Monthly quality control verification of colorimetric GP and GN identification cards was carried out for the VITEK-2-COMPACT automatic identification instrument. ATCC8739, 25923, 90028 and a blank control were used for the quality control operations on the IVD-MALDI Biotyper for each batch. Antimicrobial susceptibility tests on the strains were carried out by the disk diffusion method and the $\mathrm{E}$ test method in accordance with CLSI guidelines. Antimicrobial sensitivity test paper was purchased from OXOID Company. ATCC 25922, 25923, 27853, 90028, 35218, 700603, 29213 were used for the quality control assessment of indoor antimicrobial sensitivity tests, which were performed weekly.

Since 1998, all the data including the patient's age, sex, hospital department, hospital number, sample type, collection time, isolated pathogen(s) and drug sensitivity results have been stored in the WHONET system. Patients with incomplete data were not included in the analysis. Outpatient refers to patients with mild clinical symptoms. After a complete set of diagnostic and auxiliary examinations were conducted by the outpatient doctors, each patient was given a preliminary diagnosis. Outpatient doctors treated the patients symptomatically. When the outpatient doctors had doubts about a patient's condition or the diagnosis was that of a serious and urgent case, the patient was admitted to the inpatient ward for further examination or surgical operation in the hospital. Outpatients included general outpatients and emergency patients. Inpatients were patients who were transferred to outpatient physicians in specialist wards for further treatment according to the type and severity of the disease.

\section{Statistical analysis}

Interpretation of the antimicrobial susceptibility results was carried out in accordance with CLSI 2018 guidelines [7]. Antimicrobial sensitivity results were expressed in terms of sensitivity rates according to the CLSI M39 guidelines [8]. To avoid the effect of repetitive isolation of strains on antimicrobial sensitivity, the first isolation of strains from the same site of infection in a patient was analyzed according to CLSI M39 guidelines [8]. WHONET 5.6 software was used to analyze the antimicrobial susceptibility data. When the isolated strains were coagulase-negative staphylococcus, Corynebacterium, bacillus, Propionibacterium or other potential skin contaminants, they were considered as contaminants in this analysis. The clinical significance of a sample required two or more isolated blood cultures to confirm the diagnosis [9].

\section{Results}

From 1998 to 2017, 9130 non-intensive care unit (ICU) inpatients, 1906 ICU patients and 164 outpatient patients in Tongji Hospital had positive blood cultures. The proportion of hospitalized non-ICU patients with positive blood cultures was $81.5 \%$ (9130/11200) (Fig. 1). The most common pathogens detected were E. coli and Staphylococcus aureus from 1998 to 2017. Salmonella typhi ranked third in 1998-2002, and K. pneumoniae ranked third in 2003-2017 (Fig. 2). The limited number of strains isolated from outpatients and ICU patients

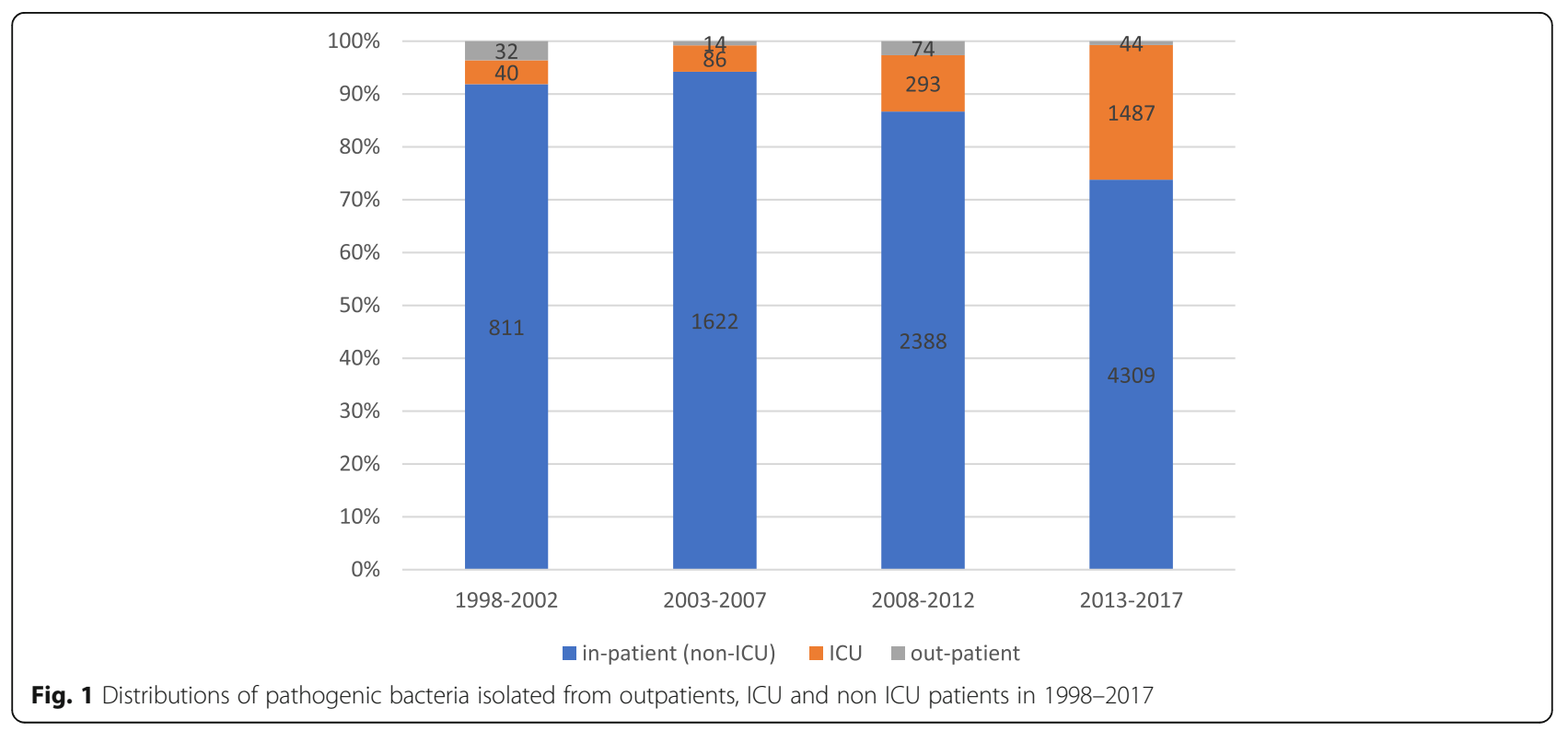




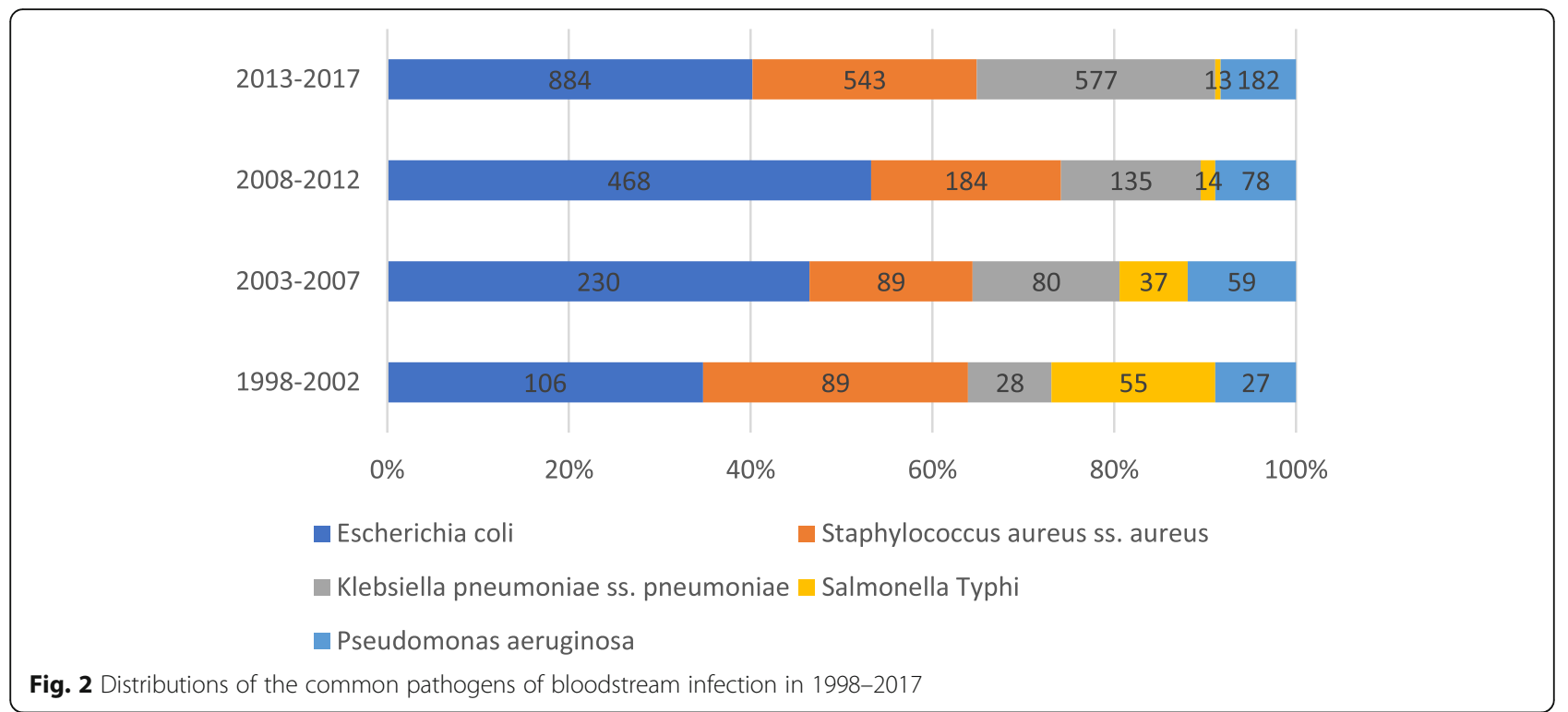

meant that only the antimicrobial sensitivity results from the hospitalized non-ICU patients were analyzed.

The antimicrobial susceptibility data showed that the susceptibility rates for E. coli to imipenem, meropenem and amikacin exceeded $90 \%$, while cefoxitin sensitivity ranged from 80 to $90 \%$. In contrast, the susceptibility rates for ampicillin and piperacillin were almost below $20 \%$. The sensitivity of $E$. coli to cefazolin, cefuroxime, cefotaxime, ceftazidime, cefepime and aztreonam showed a significant downward trend from 1998 to 2017, whereas quinolone sensitivity fluctuated slightly, and sensitivity to ciprofloxacin and levofloxacin ranged from 30 to 50\% (Fig. 3).
The sensitivity rates for $S$. aureus to vancomycin, teicoplanin and trimethoprim/sulfamethoxazole were higher than $80 \%$, while sensitivity to penicillin was lower than $10 \%$. Sensitivity to oxacillin, gentamicin and levofloxacin in Staphylococcus aureus showed a significant downward trend. Erythromycin and clindamycin sensitivity in Staphylococcus aureus fluctuated slightly, ranging from 30 to $50 \%$ and from 50 to $60 \%$, respectively (Fig. 4). The sensitivity rates for $K$. pneumoniae to imipenem, meropenem and amikacin were above $60 \%$, and this pathogen was naturally resistant to ampicillin. The changing trend of the

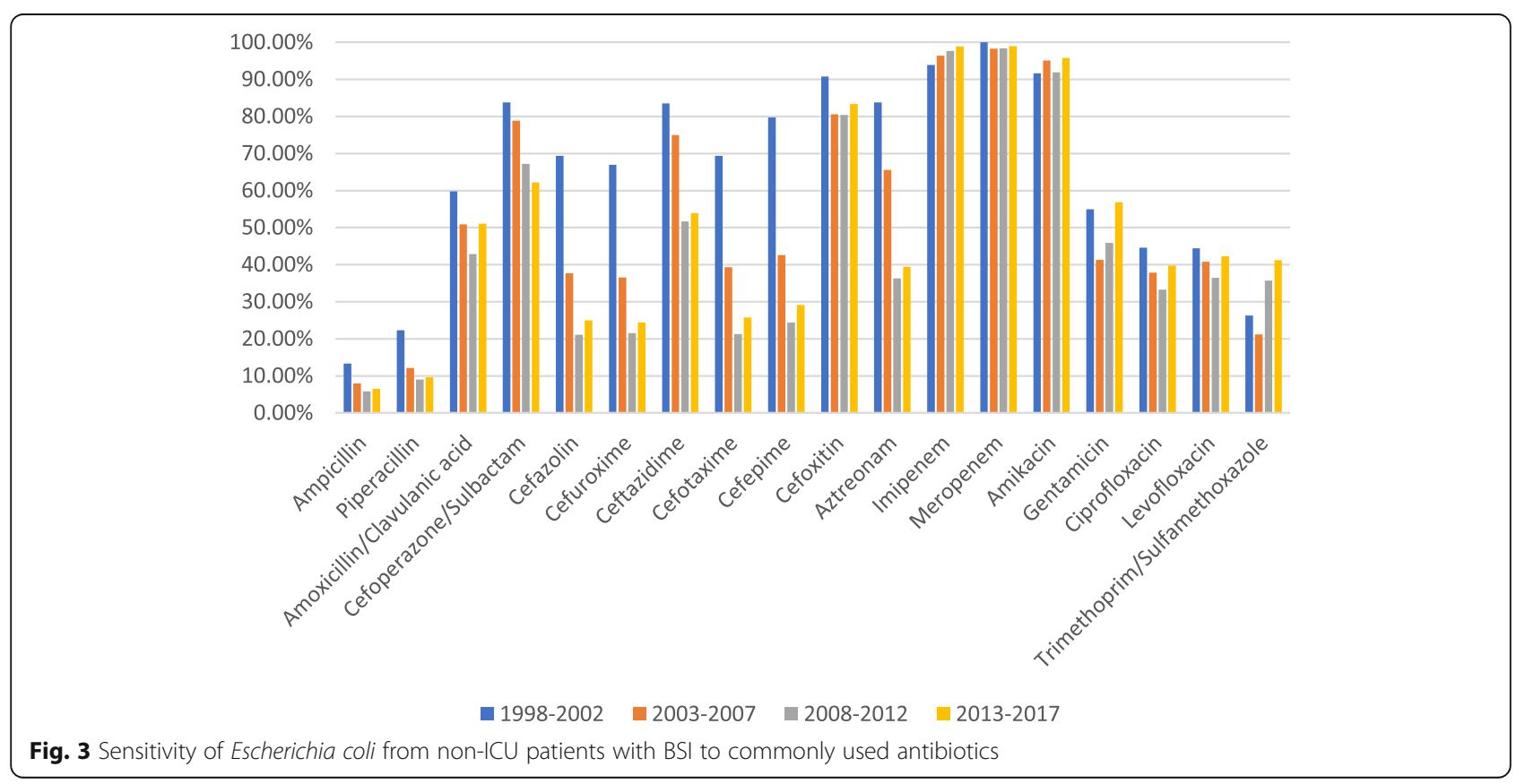




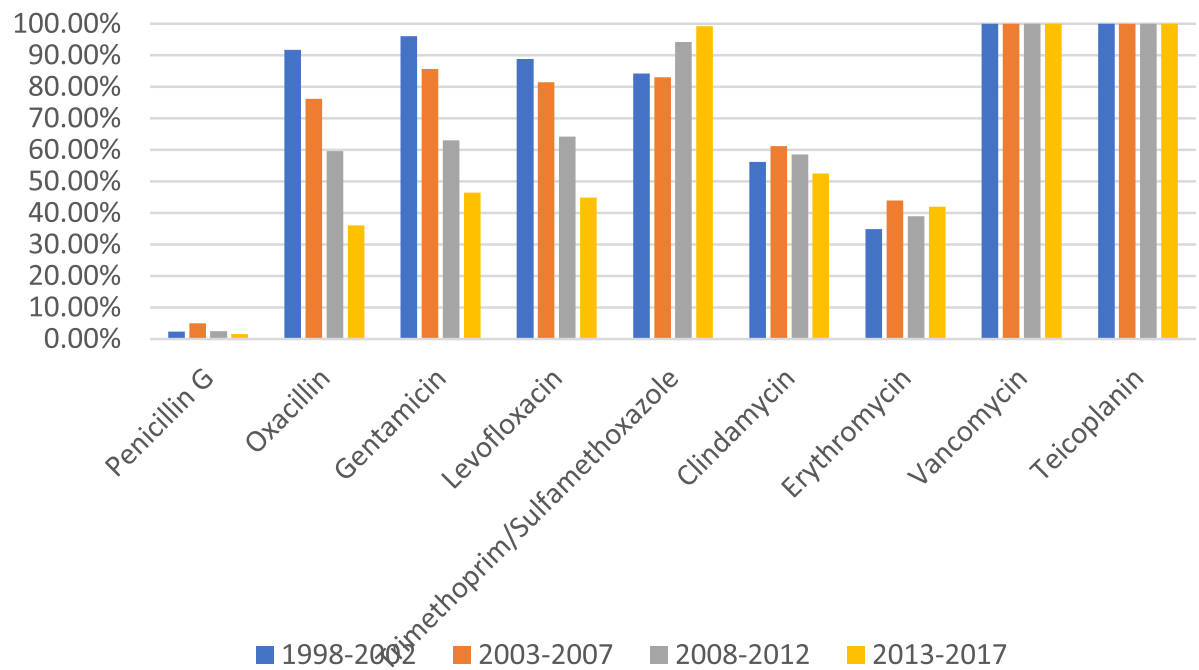

Fig. 4 Sensitivity of Staphylococcus aureus to commonly used antibiotics in patients with BSI from non-ICU

susceptibility rate, with the exception of trimetho$\mathrm{prim} / \mathrm{sulfamethoxazole}$ showed that the susceptibility rates of the other antimicrobial agents in 2013-2017 were significantly lower than those in 2008-2012 (Fig. 5). According to the changing trend for the number of multidrug resistant strains of methicillinresistantStaphylococcus aureus (MRSA), extendedspectrum beta-lactamase (ESBL)-producing $E$. coli and $K$. pneumoniae, carbapenem-resistant (CR) E. coli and $K$. pneumoniae, MRSA and CR K. pneumoniae all showed an upward trend (Fig. 6). The data for 2013 to 2017 showed that carbapenem-resistant $K$. pneumoniae in ICU patients was on the rise (Fig. 7).

\section{Discussion}

The monitoring data for Tongji Hospital from 1998 to 2017 showed that the main pathogens causing BSIs were E. coli and Staphylococcus aureus, a finding consistent with previous reports from EARS-Net, Kor-GLASS and HBARSS [2, 3, 5], but inconsistent with reports from African countries where the main BSI-causing pathogens were non-typhoidSalmonella and Salmonella typhi $[1,10]$. In our data, S. typhimurium was the third most common cause of BSIs during 1998-2002, after which it gradually decreased. Salmonella infections are food-borne diseases in which patients are mainly infected by eating Salmonella-contaminated food or drinking water [11]. Salmonella

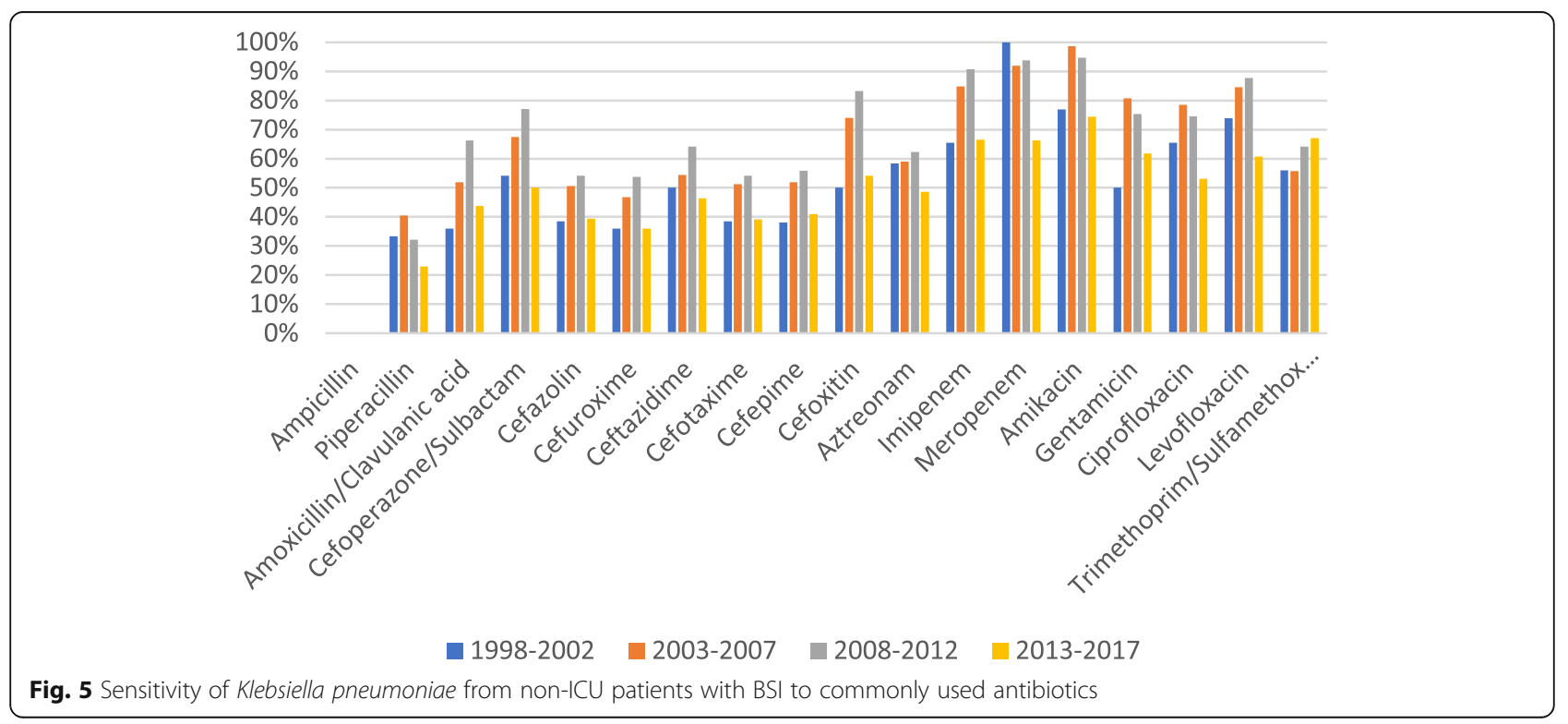




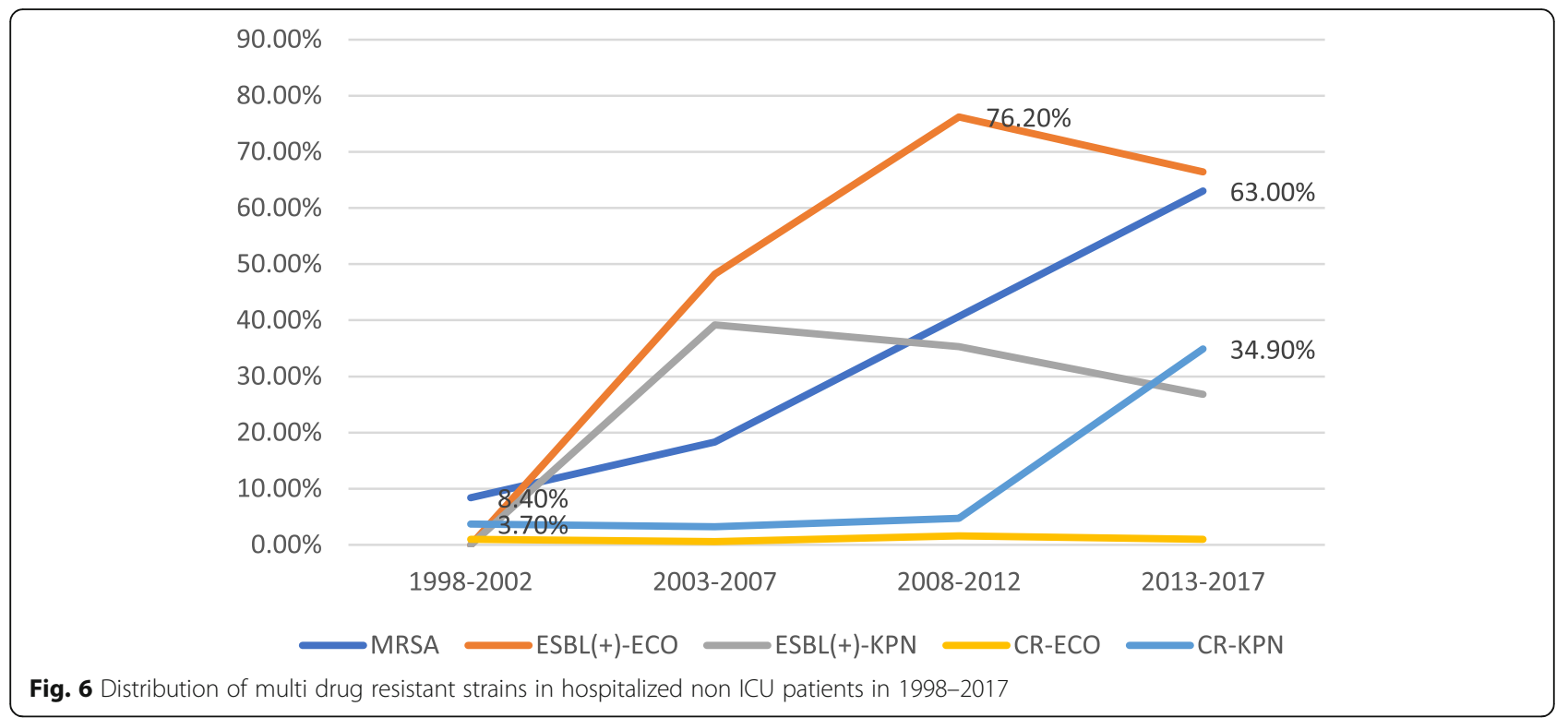

infections were severe in their clinical manifestations in African countries, and in our region from 1998 to 2002 also, which may be related to the health and medical conditions locally. Our study also found that CR K. pneumoniae, whether in ICU or non-ICU patients, significantly increased the incidence of BSIs. Data from EARS-Net showed that CR $K$. pneumoniae is a problem that should not be ignored in different European countries and in different age groups [12]. Infections caused by antibioticresistant bacteria such as MRSA, bacteria that carry ESBL genes or CR genes in K. pneumoniae and E. coli, threaten modern health care systems.

E. coli is a major cause of BSIs in Europe, and its rate of resistance to antibiotics had aroused critical concern throughout this continent [13-15]. From 1998 to 2017, we found that the susceptibility rates of $E$. coli to firstgeneration cefazolin, second-generation cefazolin and cefuroxime, third-generation cefazolin, cefotaxime and ceftazidime, fourth-generation cefazolin, cefepime and aztreonam decreased significantly in 2017 compared with 1998. Concurrently, we also found that the proportion of ESBLs produced by $E$. coli increased significantly, from $0 \%$ in $1998-2002$ to $76.2 \%$ in $2008-2012$. The rapidly increasing incidence of ESBL-expressing bacteria explains the high resistance rate of E. coli to cephalosporins and aztreonam. Whether ESBLs are associated with mortality has proved a controversial topic in different studies [16-20]. However, ESBL-producing strains can

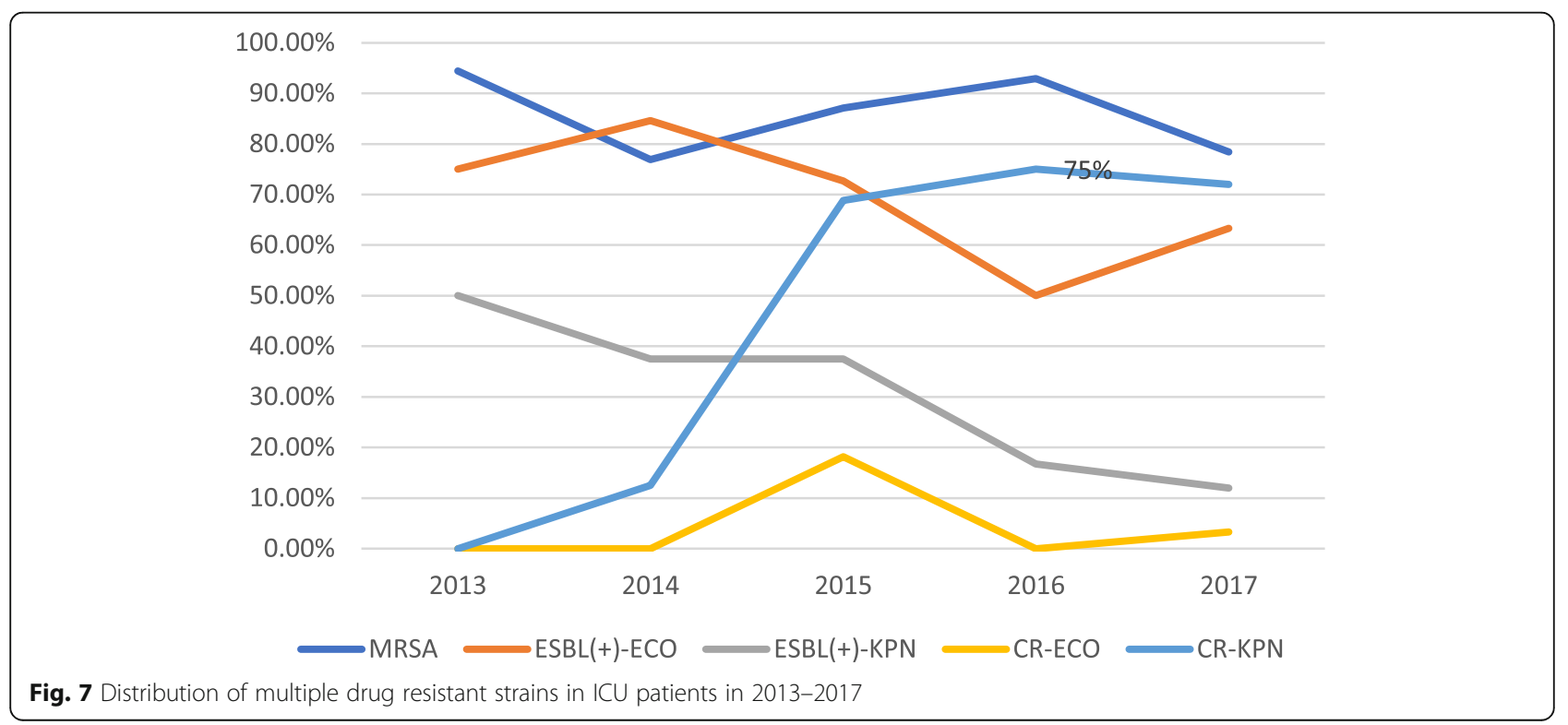


prolong hospitalization times, increase medical expenditure, and inflict higher financial burdens on patients with these infections [21, 22]. Studies have shown that the total length of hospitalization, the length of hospitalization before the infection, and the use of combined antibiotics and aminoglycosides are important risk factors for ESBL-related bloodstream infections [22].

We also found that Staphylococcus aureus was the most common causative agent of BSIs, and this bacterium was most sensitive to vancomycin and teicoplanin glycopeptide antibiotics with a sensitivity rate of $100 \%$ in 1998-2017. Trimethoprim and sulfamethoxazole were also the antibiotics that Staphylococcus aureus was most sensitive to, with a sensitivity rate above $80 \%$. However, the sensitivity of Staphylococcus aureus to levofloxacin and gentamicin declined over time. The isolation rate of MRSA increased significantly from $8.4 \%$ in 1998-2002 to $68.3 \%$ in $2013-2017$. The isolation rate of MRSA in the study region was significantly higher than that (36.6\%) reported by multi-center monitoring of 22 teaching hospitals in 12 Chinese cities in 2016 [23]. Moreover, the Chinese multicentric monitoring data for 2013 to 2016 and the data for Germany in 2014 showed that the sensitivity of MRSA to quinolones was increasing, but our data showed a decline [23, 24]. The possible reasons underlying this finding are related to the extensive use of quinolones and aminoglycosides in our study region. The data for 22 Hong Kong hospitals showed that the 30-day mortality rate for patients with MRSAinduced BSIs was $32.39 \%$ [25], revealing that MRSArelated BSIs were a significant burden in Hong Kong [25], as they were also in our study area.

It has been found that after E. coli, K. pneumoniae is the predominant BSI-causing pathogen [26-28], and CR is the most serious problem in BSIs caused by it. In the present study, the detection rate for CR K. pneumoniae in non-ICU patients increased from 3.7\% in 1998-2002 to $34.9 \%$ in $2013-2017$. In ICU patients, the detection rate for CR K. pneumoniae increased from 0\% in 2013 to $75 \%$ in 2016. Consistent with this finding, the surveillance data from CHINET in China showed that CR $K$. pneumoniae increased significantly from 2005 to 2014 [29]. Carbapenems have been used as the first-line antibiotics for treating multidrug-resistant $K$. pneumoniae [30]. However, resistance to carbapenems is making the treatment of CR K. pneumoniae extremely difficult. The results of a retrospective study conducted at a Shanghai teaching hospital revealed an overall 30-day mortality rate for BSIs of $25 \%$ for patients who had contracted CR K. pneumoniae [31]. Another retrospective cohort study on the effect of appropriate combination therapy on mortality in patients with BSIs caused by carbapenemaseproducingEnterobacteriaceae reported that use of combination therapy was associated with improved survival only in patients with a high mortality risk score [32]. The authors also reported that patients with BSIs caused by carbapenemase-producingEnterobacteriaceae should receive active therapy upon diagnosis, and monotherapy should be considered for those in the low mortality-score stratum [32].

The present study is the first report on the accumulated data from our center at Tongji Hospital covering the past 20 years. Our findings augment current understanding on the pathogenic bacteria responsible for BSIs and the changes in drug resistance occurring in them over time in central China.

\section{Conclusions}

The present focus of bacterial resistance control is to curb the spread of multi-drug resistant strains, especially CR $K$. pneumoniae, the infectious from which have increased significantly in recent years, thereby meriting special attention. Finally, controlling infections with MRSA remains an important goal.

\section{Abbreviations}

BSIs: Bacterial bloodstream infections; CAP: College of American Pathologists: CLSI: Clinical \& Laboratory Standards Institute; CR: carbapenem-resistant; EARS-Net: European Antimicrobial Resistance Surveillance Network; ESBL: extended-spectrum beta-lactamase; HBARSS: Hubei Province Antimicrobial Resistance Surveillance System; ICUs: intensive care units; MRSA: methicillin-resistant Staphylococcus aureus; WBC: white blood cell

\section{Acknowledgments}

We thank all members of laboratory medicine department of TongJi hospital for their participation in these studies. We thank Sandra Cheesman, PhD, from Liwen Bianji, Edanz Group China (www.liwenbianji.cn/ac), for editing the English text of a draft of this manuscript.

\section{Authors' contributions}

ZS designed the study. LT and ZZ analyzed the data. LT wrote the article. All authors reviewed the manuscript prior to submission. All authors read and approved the final manuscript.

\section{Funding}

This work was supported by research grants from the National Mega Project on Major Infectious Disease Prevention (2017ZX10103005-007). The funders had no role in study design, data collection and analysis, decision to publish, or preparation of the manuscript.

\section{Availability of data and materials}

The datasets used and/or analyzed during the current study are available from the corresponding author on reasonable request.

\section{Ethics approval and consent to participate}

The study protocol was approved by the Tongji Hospital ethics committee for research in health. The Tongji Hospital ethics committee also approved the waiver of informed consent to participate in this study due to its retrospective design. All patient data were anonymous prior to the analysis.

\section{Consent for publication}

Not applicable.

\section{Competing interests}

The authors declare that they have no competing interest.

\section{Author details}

'Department of Clinical Laboratory, Tongji Hospital, Tongji Medical College, Huazhong University of Science and Technology, Wuhan, Hubei Province, 
China. ${ }^{2}$ Department of Pharmacy, Tongji Hospital, Tongji Medical College, Huazhong University of Science and Technology, Wuhan, Hubei Province, China.

Received: 21 February 2019 Accepted: 21 May 2019

Published online: 28 May 2019

\section{References}

1. Musicha P, Cornick JE, Bar-Zeev N, French N, Masesa C, Denis B, Kennedy N, Mallewa J, Gordon MA, Msefula CL, et al. Trends in antimicrobial resistance in bloodstream infection isolates at a large urban hospital in Malawi (19982016): a surveillance study. Lancet Infect Dis. 2017;17(10):1042-52.

2. Gagliotti C, Balode A, Baquero F, Degener J, Grundmann H, Gur D, Jarlier V, Kahlmeter G, Monen J, Monnet DL, et al. Escherichia coli and Staphylococcus aureus: bad news and good news from the European antimicrobial resistance surveillance network (EARS-net, formerly EARSS), 2002 to 2009. Euro Surveill. 2011;16(11).

3. Lee H, Yoon EJ, Kim D, Jeong SH, Won EJ, Shin JH, Kim SH, Shin JH, Shin KS, Kim YA, et al. Antimicrobial resistance of major clinical pathogens in South Korea, may 2016 to April 2017: first one-year report from Kor-GLASS. Euro Surveill. 2018;23(42).

4. Hattori H, Maeda M, Nagatomo Y, Takuma T, Niki Y, Naito Y, Sasaki T, Ishino K. Epidemiology and risk factors for mortality in bloodstream infections: a single-center retrospective study in Japan. Am J Infect Control. 2018.

5. Tian $L$, Sun Z, Zhang Z. Antimicrobial resistance of pathogens causing nosocomial bloodstream infection in Hubei Province, China, from 2014 to 2016: a multicenter retrospective study. BMC Public Health. 2018;18(1):1121.

6. Health and Family Planning Commission. Operating procedures of blood culture for clinical microbiology laboratory. WS/T 503, GB,GBT,GB/T: Chinese Standard; 2017

7. Clinical and Laboratory Standards Institute. Performance Standards for Antimicrobial Susceptibility Testing, Twenty-eighth Informational Supplement, M100-S28. Wayne, PA: Clin Lab Stand Institute; 2018.

8. Clinical and Laboratory Standards Institute. Analysis and presentation of cumulative antimicrobial susceptibility test data, M39-A4. Wayne, PA: Clin Lab Stand Institute; 2014

9. Weinstein MP, Murphy JR, Reller LB, Lichtenstein KA. The clinical significance of positive blood cultures: a comprehensive analysis of 500 episodes of bacteremia and fungemia in adults. II. Clinical observations, with special reference to factors influencing prognosis. Rev Infect Dis. 1983;5(1):54-70.

10. Eibach D, Belmar Campos C, Krumkamp R, Al-Emran HM, Dekker D, Boahen KG, Kreuels B, Adu-Sarkodie Y, Aepfelbacher M, Park SE, et al. Extended spectrum beta-lactamase producing Enterobacteriaceae causing bloodstream infections in rural Ghana, 2007-2012. Int J Med Microbiol. 2016;306(4):249-54.

11. Galanis E, Lo Fo Wong DM, Patrick ME, Binsztein N, Cieslik A, Chalermchikit T, Aidara-Kane A, Ellis A, Angulo FJ, Wegener HC, et al. Web-based surveillance and global Salmonella distribution, 2000-2002. Emerg Infect Dis. 2006;12(3):381-8.

12. Cassini A, Hogberg LD, Plachouras D, Quattrocchi A, Hoxha A, Simonsen GS, Colomb-Cotinat M, Kretzschmar ME, Devleesschauwer B, Cecchini M, et al. Attributable deaths and disability-adjusted life-years caused by infections with antibiotic-resistant bacteria in the EU and the European economic area in 2015: a population-level modelling analysis. Lancet Infect Dis. 2018.

13. Vihta KD, Stoesser N, Llewelyn MJ, Quan TP, Davies T, Fawcett NJ, Dunn L, Jeffery K, Butler CC, Hayward G, et al. Trends over time in Escherichia coli bloodstream infections, urinary tract infections, and antibiotic susceptibilities in Oxfordshire, UK, 1998-2016: a study of electronic health records. Lancet Infect Dis. 2018;18(10):1138-49.

14. Buetti N, Atkinson A, Marschall J, Kronenberg A. Swiss Centre for Antibiotic $R$ : incidence of bloodstream infections: a nationwide surveillance of acute care hospitals in Switzerland 2008-2014. BMJ Open. 2017;7(3):e013665.

15. van der Mee-Marquet NL, Blanc DS, Gbaguidi-Haore H, Dos Santos Borges S, Viboud Q, Bertrand X, Quentin R. Marked increase in incidence for bloodstream infections due to Escherichia coli, a side effect of previous antibiotic therapy in the elderly. Front Microbiol. 2015;6:646.

16. Schwaber MJ, Carmeli Y. Mortality and delay in effective therapy associated with extended-spectrum beta-lactamase production in Enterobacteriaceae bacteraemia: a systematic review and meta-analysis. J Antimicrob Chemother. 2007;60(5):913-20.
17. Lin YT, Liu CJ, Fung CP, Tzeng CH. Nosocomial Klebsiella pneumoniae bacteraemia in adult cancer patients--characteristics of neutropenic and non-neutropenic patients. Scand J Infect Dis. 2011;43(8):603-8.

18. Nasa $P$, Juneja $D$, Singh $O$, Dang $R$, Singh $A$. An observational study on bloodstream extended-spectrum beta-lactamase infection in critical care unit: incidence, risk factors and its impact on outcome. Eur J Intern Med. 2012;23(2):192-5.

19. Ben-David D, Kordevani R, Keller N, Tal I, Marzel A, Gal-Mor O, Maor Y, Rahav G. Outcome of carbapenem resistant Klebsiella pneumoniae bloodstream infections. Clin Microbiol Infect. 2012;18(1):54-60.

20. Gurntke S, Kohler C, Steinmetz I, Pfeifer Y, Eller C, Gastmeier P, Schwab F, Leistner R. Molecular epidemiology of extended-spectrum beta-lactamase (ESBL)-positive Klebsiella pneumoniae from bloodstream infections and risk factors for mortality. J Infect Chemother. 2014;20(12):817-9.

21. Leistner R, Gurntke S, Sakellariou C, Denkel LA, Bloch A, Gastmeier P, Schwab F. Bloodstream infection due to extended-spectrum beta-lactamase (ESBL)-positive K. pneumoniae and E. coli: an analysis of the disease burden in a large cohort. Infection. 2014;42(6):991-7.

22. Tanir Basaranoglu S, Ozsurekci Y, Aykac K, Karadag Oncel E, Bicakcigil A, Sancak B, Cengiz AB, Kara A, Ceyhan M. A comparison of blood stream infections with extended spectrum beta-lactamase-producing and non-producing Klebsiella pneumoniae in pediatric patients. Ital J Pediatr. 2017:43(1):79.

23. Li S, Sun S, Yang C, Chen H, Yin Y, Li H, Zhao C, Wang $H$. The changing pattern of population structure of Staphylococcus aureus from bacteremia in China from 2013 to 2016: ST239-030-MRSA replaced by ST59-t437. Front Microbiol. 2018:9:332.

24. Schaumburg F, Idelevich EA, Peters G, Mellmann A, von Eiff C, Becker K. Study G: trends in antimicrobial non-susceptibility in methicillin-resistant Staphylococcus aureus from Germany (2004-2011). Clin Microbiol Infect. 2014;20(9):O554-7.

25. You JHS, Choi KW, Wong TY, Ip M, Ming WK, Wong RY, Chan SN, Tse HT, Chau CTS, Lee NLS. Disease burden, characteristics, and outcomes of methicillin-resistant Staphylococcus aureus bloodstream infection in Hong Kong. Asia Pac J Public Health. 2017;29(5):451-61.

26. Pitout JD, Laupland KB. Extended-spectrum beta-lactamase-producing Enterobacteriaceae: an emerging public-health concern. Lancet Infect Dis. 2008;8(3):159-66.

27. Ardanuy C, Linares J, Dominguez MA, Hernandez-Alles S, Benedi VJ, Martinez-Martinez L. Outer membrane profiles of clonally related Klebsiella pneumoniae isolates from clinical samples and activities of cephalosporins and carbapenems. Antimicrob Agents Chemother. 1998;42(7):1636-40.

28. Uslan DZ, Crane SJ, Steckelberg JM, Cockerill FR 3rd, St Sauver JL, Wilson WR, Baddour LM. Age- and sex-associated trends in bloodstream infection: a population-based study in Olmsted County, Minnesota. Arch Intern Med. 2007;167(8):834-9.

29. Hu FP, Guo Y, Zhu DM, Wang F, Jiang XF, Xu YC, Zhang XJ, Zhang CX, Ji P, $X i e Y$, et al. Resistance trends among clinical isolates in China reported from CHINET surveillance of bacterial resistance, 2005-2014. Clin Microbiol Infect. 2016;22(Suppl 1):S9-14.

30. Bradford PA, Urban C, Mariano N, Projan SJ, Rahal JJ, Bush K. Imipenem resistance in Klebsiella pneumoniae is associated with the combination of ACT-1, a plasmid-mediated AmpC beta-lactamase, and the Foss of an outer membrane protein. Antimicrob Agents Chemother. 1997;41(3):563-9.

31. Li L, Huang H. Risk factors of mortality in bloodstream infections caused by Klebsiella pneumonia: a single-center retrospective study in China. Medicine (Baltimore). 2017;96(35):e7924.

32. Gutierrez-Gutierrez B, Salamanca E, de Cueto M, Hsueh PR, Viale P, Pano-Pardo JR, Venditti M, Tumbarello M, Daikos G, Canton R, et al. Effect of appropriate combination therapy on mortality of patients with bloodstream infections due to carbapenemase-producing Enterobacteriaceae (INCREMENT): a retrospective cohort study. Lancet Infect Dis. 2017;17(7):726-34.

\section{Publisher's Note}

Springer Nature remains neutral with regard to jurisdictional claims in published maps and institutional affiliations. 